\title{
Original
}

\section{Incidencia de notificación de situaciones conflictivas con los ciudadanos en el área 6 de la comunidad autónoma de Madrid}

\author{
Incidence of notification of conflict situations with citizens \\ in area 6 of the community of Madrid
}

\section{María Maestre Naranjo, Jenry Borda Olivas, Arantxa Ortega Hernández, Coral Sainz Pinós, Belén Cabrero Martín, M. ${ }^{a}$ Luisa Rodriguez de la Pinta}

Servicio de Prevención. Hospital Universitario Puerta de Hierro. Majadahonda (Madrid). España.

\section{Recibido: 09-06-11 \\ Aceptado: 13-06-11}

\section{Correspondencia}

María Maestre Naranjo

Hospital Universitario Puerta de Hierro Majadahonda

C/.Joaquín Rodrigo, 2

28222 Majadahonda (Madrid). España.

Tfno: 911917414

E-mail: mmaestre.hpth@salud.madrid.org

\section{Resumen}

La violencia en el lugar de trabajo se ha convertido en un problema mundial; afectando a todos los sectores y categorías laborales.

Las consecuencias de la violencia en las victimas generan enfermedad física, problemas psicológicos, abuso de medicamentos y sustancias tóxicas; también, se ven afectados el ámbito familiar y social.

Se realiza un estudio en los trabajadores del SERMAS (Servicio Madrileño de Salud) del área 6 de salud de la Comunidad Autónoma de Madrid, para determinar la incidencia de la comunicación de situaciones conflictivas con los ciudadanos, identificar el ámbito de actuación, la categoría profesional más afectada, conocer la prevalencia del tipo de agresión e identificar las consecuencias tras las situaciones conflictivas notificadas.

Med Segur Trab (Internet) 2011; 57 (223) 134-146

Palabras clave: situacion conflictiva, notificación, violencia en el trabajo, consecuencias tras las situaciones conflictivas notificadas.

Abstract

Violence in the workplace has become a global problem, affecting all sectors and job categories.

The consequences of violence on victims generate physical illness, psychological problems, drug and toxic substances abuse. Family and social sphere are also affected.

We performed a study to determine the incidence of the communication of conflict situations between citizens and SERMAS workers (Madrid Health Service) in area 6 of the Community of Madrid, as well as to 
identify the scope, identify job categories most affected, discover the prevalence of the type of aggression and identify the consequences after reporting conflict situations.

Med Segur Trab (Internet) 2011; 57 (223) 134-146

Key Word: conflict situations, notification, violence in the workplace, consequences after reporting conflict situations. 


\section{INTRODUCCIÓN}

La violencia en el lugar de trabajo se ha convertido en un problema mundial; afectando a todos los sectores y categorías laborales ${ }^{1,2}$. Es un fenómeno emergente y global, cuya prevención y manejo exige una actuación coordinada de todas las instituciones implicadas.

Según Nordin, la violencia en el lugar de trabajo afecta especialmente a los trabajadores de la salud (el $25 \%$ de todos los incidentes violentos se registran en este sector $)^{3}$. Son casos de violencia física o psicológica predominando esta sobre la primera. En muchas ocasiones los actos de violencia no se denuncian y no se registran, por lo que desconocemos la verdadera magnitud del problema.

La OMS dice "la violencia en el lugar de trabajo, no es un problema individual ni aislado, es un problema estructural y estratégico que tiene sus raíces en factores sociales, económicos, organizativos y culturales" por lo que debe recibir un tratamiento integral. ${ }^{2}$

La OIT y la OMS proponen una definición de violencia adaptada por la Comisión Europea, "la violencia laboral está constituida por incidentes en los que el trabajador sufre malos tratos, amenazas o ataques en circunstancias relacionadas con su trabajo y ponen en peligro, implícita o explícitamente, su seguridad, su bienestar o su salud"

Las consecuencias de la violencia en las victimas generan sentimientos de cólera, ira, pérdida de confianza en sí mismo y desconfianza hacia las personas extrañas. Si la situación se prolonga, las consecuencias pueden producir enfermedad física o problemas psicológicos, abuso de medicamentos y sustancias tóxicas; también, se van a ver afectados el ámbito familiar y social y puede llevarles al abandono de la profesión. , 5, 6

En las empresas: la violencia conduce a un empeoramiento de los resultados, altera las relaciones interpersonales y el clima laboral. Aumenta el absentismo, la rotación de los trabajadores, disminuyendo la eficacia y la productividad. ${ }^{6,7,8}$

Estas situaciones surgen en la mayoría de los casos cuando los pacientes ven frustradas sus expectativas respecto al sistema sanitario, ya que estas son más exigentes que la oferta real del sistema. ${ }^{7,8}$

Las agresiones a los profesionales del ámbito sanitario, también ocurren en otros países tanto de nuestro entorno como puede ser Francia y el Reino Unido, como fuera del mismo (Estados Unidos, Canadá, Australia y Nueva Zelanda). ${ }^{1,9}$

En muchos países europeos se ha optado por la vía legislativa para afrontar estas situaciones, debido al número y gravedad de los casos. ${ }^{9}$

En nuestro país colegios profesionales como el Colegio Oficial de Médicos de Barcelona ${ }^{10}$, el Colegio de Médicos de León y las Comunidades Autónomas como Valencia, Castilla la Mancha, Andalucía y Baleares han puesto en marcha registros de agresiones para el personal médico.

Hay comunidades autónomas que regulan estas situaciones a través de distintas normativas, es el caso de las Comunidades Autónomas de Castilla La Mancha, Andalucía, y Murcia entre otras.

La Consejería de Sanidad de la Comunidad de Madrid, reconociendo el progresivo incremento de agresiones a los profesionales sanitarios, publica la Orden 212/2004 por la que establece las directrices para la elaboración de planes de prevención y atención frente a potenciales situaciones conflictivas con los ciudadanos ${ }^{11}$.

Posteriormente ha sido modificada por la Orden 683/2006 por la que se establecen las directrices y líneas generales para la elaboración de Planes de Prevención y Atención frente a situaciones conflictivas con los ciudadanos en los centros e instituciones sanitarias y crea la Comisión Central de Seguimiento. ${ }^{12}$ 
En el año 2008, a través de la Orden 22/2008, de 21 de enero, se crea el fichero de datos de carácter personal denominado plan regional de Prevención de situaciones conflictivas dependiente de la Dirección General de RRHH de la Consejería de Sanidad de la Comunidad de Madrid ${ }^{13}$

Recientemente a través de la Resolución de la Dirección General de Recursos Humanos de 29 de enero de 2009, se dictan instrucciones para la puesta en marcha del. registro cenralizado de situaciones conflictivas ${ }^{14}$

Los estudios realizados en nuestro país en relación con este tema son los siguientes:

El trabajo, becado por la Fundación Prevent durante el ejercicio 2006, expone el diseño y los resultados de la implementación en diversos centros sanitarios de Cataluña a través de la web "www.violenciaocupacional.net", de un sistema informatizado de Notificación on-line de Incidentes Violentos. ${ }^{15}$

La información sobre la violencia ocupacional en el sector de la salud se obtenía básicamente de encuestas de autoinforme, con bajos porcentajes de respuesta y notables problemas metodológicos de validez, por los diversos sesgos que conlleva. Este proyecto tiene como objetivo diseñar planes de mejora, tanto en la prevención primaria como en las actuaciones post incidente. Describe la tipología y la prevalencia de esta violencia en el colectivo investigado, identificando los principales factores de riesgo de la misma y propone elementos para su prevención.

Informe realizado por la UNIVERSIDAD de ZARAGOZA, siendo publicado ${ }^{16}$. "Aggression Towads Health Care Workers in Spain: A Multi-facility Study to Evaluate the Distribution of Growing Violence Among Professionals, Health Facilitgies and Departaments (Gascón, S: Martínez-Jarreta, B; Santed, M.A: González Andrade, J.F. y Casolod, Y) International Journal of Occupational and Environmetal Health (Volumen 15, n. ${ }^{\circ} 1$ [2009]). ${ }^{16}$

\section{OBJETIVOS}

Dada la repercusión que estos incidentes pueden tener sobre los trabajadores ${ }^{4,5,6}$ es importante conocer en qué situación se encuentran los profesionales sanitarios de nuestra área de salud, para identificar a los grupos de población sanitaria más vulnerables a las situaciones conflictivas con los ciudadanos en función de su categoría laboral, ámbito de trabajo (atención primaria o especializada) que desarrollan sus tareas para poder distribuir, adecuadamente, los recursos, en función de las necesidades observadas tras el análisis de los resultados obtenidos. Así mismo pueden ser el primer paso para la instauración de medidas preventivas y la identificación de posibles factores de riesgo que conducen al ciudadano a estas actuaciones.

Nos planteamos los siguientes objetivos:

1. Determinar la incidencia de la comunicación de situaciones conflictivas con los ciudadanos en los trabajadores del SERMAS (Servicio Madrileño de Salud) de nuestra área de salud, como objetivo principal.

2. Identificar el ámbito de actuación más frecuente en el que ocurren estos incidentes.

3. Identificar las categorías laborales más afectadas.

4. Conocer la incidencia del tipo de agresión.

5. Identificar las consecuencias tras las situaciones conflictivas notificadas. 


\section{METODOLOGÍA}

Estudio descriptivo transversal, en los trabajadores del Servicio Madrileño de Salud (SERMAS) del Área 6 de la Comunidad Autónoma de Madrid (CAM), durante un período de 15 meses (2 de febrero del 2009 al 30 de abril del 2010).

La CAM a través de los Servicios de Prevención ubicados en las Áreas Sanitarias es la responsable de implantar las medidas preventivas destinadas a disminuir las situaciones conflictivas con los ciudadanos en los centros de trabajo del sector sanitario, siendo una de estas actividades el registro, seguimiento y asistencia de las situaciones conflictivas con los ciudadanos notificadas por los trabajadores, así como la elaboración de los planes de prevención ante estos incidentes ${ }^{12}$

El estudio se realiza mediante el Registro de las Situaciones Conflictivas con los usuarios, notificadas por los trabajadores del SERMAS del Área 6 de la CAM, tras la implantación de la Resolución de la Dirección General de Recursos Humanos de 29 de enero del $2009^{14}$

La fecha de inicio de la aplicación y por tanto del formulario de comunicación de las agresiones por parte de los trabajadores, corresponde al día 2 de febrero de 2009.

Ese mismo día se puso a disposición de cada Gerencia y Servicio de Prevención en la intranet de la Consejería (http://saluda.salud.madrid.org), el formulario único de registro del incidente que unifica y sustituye a los diferentes modelos existentes en los centros sanitarios para comunicar este tipo de situaciones.

La aplicación es accesible, únicamente, desde los centros sanitarios que se encuentren dentro de la red de la Consejería de Sanidad de la Comunidad de Madrid, a través de la página web.

El trabajador tiene acceso al formulario de notificación de situaciones conflictivas con los ciudadanos así como a las instrucciones para su cumplimentación en la intranet de la consejería http://saluda.salud.madrid.org, en las jefaturas y supervisiones de enfermería de los servicios y unidades de los hospitales, o bien en el portal interno del área para los trabajadores de Atención Primaria. ${ }^{14}$

Los trabajadores comunican cualquier situación conflictiva a través de dicho formulario que una vez cumplimentado y firmado deberá ser remitido a la Gerencia correspondiente, quien enviará copia al Servicio de Prevención, a la mayor brevedad posible, trasladando esta información al Registro Centralizado de la Consejería de Sanidad, (anexo I)

La población estudiada son los 5726 trabajadores del Área 6 de la CAM. El área geográfica corresponde a una superficie de $1382 \mathrm{Km}^{2}$, con una densidad de población de 301 habitantes $/ \mathrm{km}^{2}$, atendiendo a una población de 415.967 personas, (hombres: 201.832; mujeres: 214.315) (anexo II).

Asumimos que no toda la población incluida en el estudio tiene la misma incidencia de situaciones conflictivas con los ciudadanos, como son los jefes de servicio, supervisoras de enfermería y los trabajadores de las direcciones correspondientes.

Las variables a estudio se obtienen de los ítems recogidos en el cuestionario de notificación de situaciones conflictivas con los ciudadanos. Las clasificamos en variables relacionadas con el trabajador (ámbito de actuación, centro de trabajo y categoría laboral), variables relacionadas con el incidente (tipo de agresión: física o psíquica) y variables relacionadas con las consecuencias del incidente (baja laboral, lesiones físicas o psíquicas).

Según el ámbito de actuación se clasifica en Atención Primaria (AP) y Atención Especializada (AE). Los centros de trabajo de Atención Especializada son: el Hospital Universitario Puerta de Hierro Majadahonda, Hospital El Escorial y Hospital Guadarrama, unificando la diversidad de centros de Atención Primaria en rural y urbano.

Según la categoría laboral los trabajadores pueden ser personal sanitario y no sanitario. El personal sanitario engloba a los facultativos, residentes y al personal de 
enfermería (enfermeras, fisioterapeutas, matronas y técnicos). El personal no sanitario (celadores, administrativos, trabajadores sociales y otros como personal de limpieza, capellán, telefonista, técnicos de control).

Se excluyen a 598 trabajadores no sanitarios que realizan sus tareas en el hospital Universitario Puerta de Hierro Majadahonda por no ser trabajadores del Servicio Madrileño de Salud y pertenecer a empresas subcontratadas que prestan sus servicios en el mismo. Se excluyen así mismo aquellos trabajadores que por las tareas que realizan no tienen relación directa con los ciudadanos como personal de mantenimiento, personal de cocina, determinado grupo de administrativos y celadores (personal, laboratorios, biblioteca, escuelas), personal de enfermería y facultativos de laboratorios (anatomía patológica, microbiología, bioquímica, inmunología), siendo la población definitiva del estudio de 4842 sujetos. (Tabla 1)

Las categorías laborales varían en función del ámbito de actuación y del centro de trabajo debido a las características asistenciales de los mismos y el tipo de relación laboral de los trabajadores con la empresa (Consejería de Sanidad).

Tabla 1. Diagrama de flujo con el número de trabajadores del Área 6 de la CAM

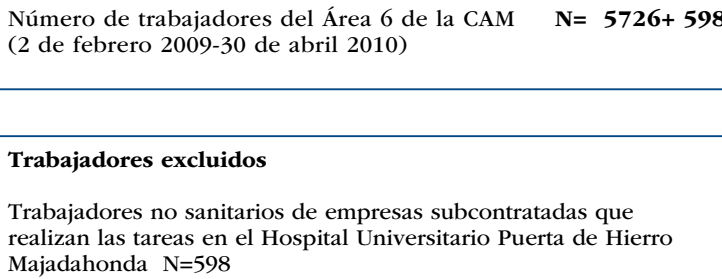

Trabajadores no sanitarios de empresas subcontratadas que realizan las tareas en el Hospital Universitario Puerta de Hierro Majadahonda $\mathrm{N}=598$

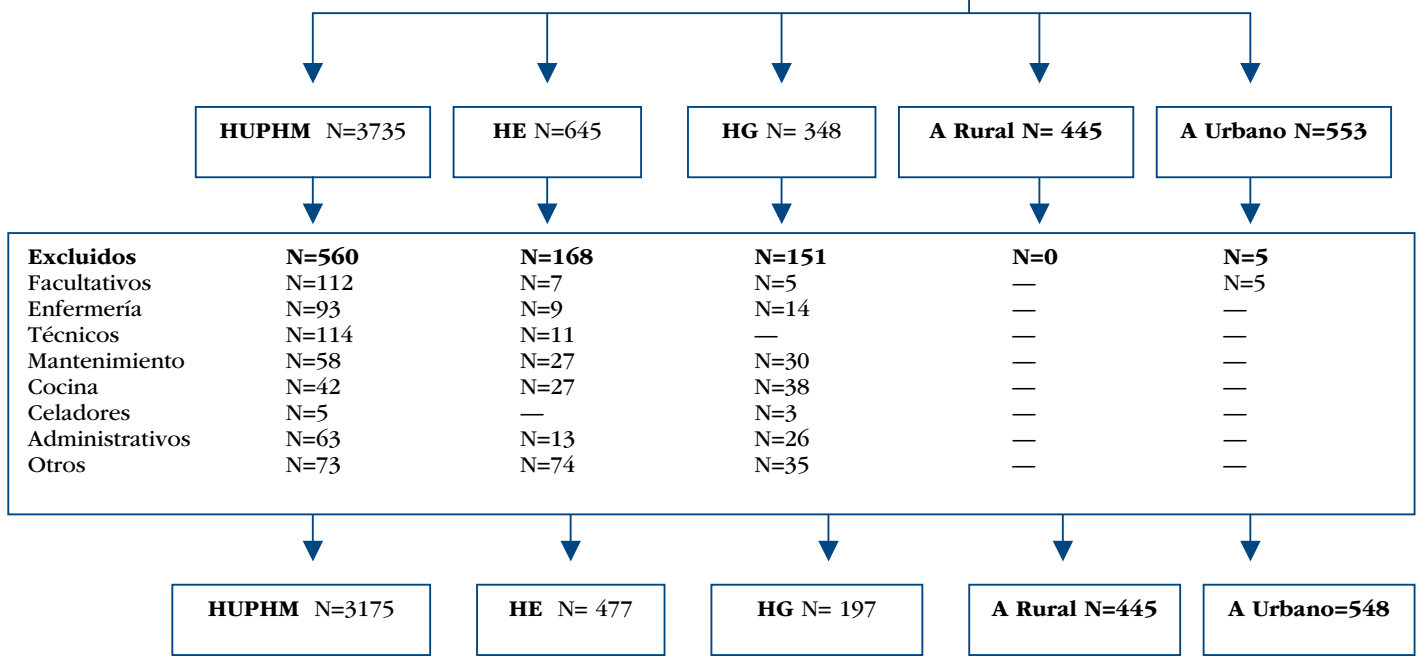

El número de trabajadores a estudio en función de sus categorías laborales, ámbito de actuación y centros de trabajo quedan reflejados en la (Tabla 2) 
Tabla 2. (Población a estudio)

\begin{tabular}{|c|c|c|c|c|c|c|}
\hline & & & & & & \\
\hline & & \multicolumn{5}{|c|}{ ÁREA 6} \\
\hline & & \multicolumn{3}{|c|}{ ATENCIÓN ESPECIALIZADA } & \multicolumn{2}{|c|}{ ATENCIÓN PRIMARIA } \\
\hline & & HUPHM & HE & HG & Ámbito Rural & Ámbito Urbano \\
\hline \multirow{4}{*}{$\begin{array}{l}\text { Personal } \\
\text { Sanitario }\end{array}$} & Facultativos & 970 & 102 & 10 & 177 & 219 \\
\hline & Enfermeras & 1746 & 278 & 134 & 151 & 191 \\
\hline & Fisioterapeutas & 32 & - & 18 & 7 & 8 \\
\hline & Técnicos & 114 & 16 & 3 & - & - \\
\hline \multirow{3}{*}{$\begin{array}{l}\text { Personal } \\
\text { no Sanitario }\end{array}$} & Celadores & 135 & 27 & 20 & 17 & 22 \\
\hline & Administrativos & 167 & 54 & 12 & 88 & 103 \\
\hline & Higienista dental & - & - & - & 5 & 5 \\
\hline Total & 4842 & 3175 & 477 & 197 & 445 & 548 \\
\hline \multicolumn{7}{|c|}{$\begin{array}{l}\text { HUPHM: Hospital Universitario Puerta de Hierro Majadahonda } \\
\text { HE: Hospital El Escorial } \\
\text { HG: Hospital Guadarrama }\end{array}$} \\
\hline
\end{tabular}

Las variables según el tipo de agresión: agresión física, agresión verbal (amenazas, coacciones, insultos, injurias y vejaciones).

Las variables relacionadas con las consecuencias del incidente en función de la existencia de lesiones (físicas o psíquicas), si precisan o no apoyo psicologico, y si el incidente ha conducido a una baja laboral que es considerada como accidente de trabajo.

\section{ANÁLISIS ESTADÍSTICO}

Se ha realizado mediante el programa estadístico SPSS versión 14.0, efectuando una descripción de frecuencias absolutas y relativas de las variables a estudio, comparando los porcentajes con el test de chi cuadrado de Pearson y chi cuadrado corregido por continuidad de las variables.

\section{RESULTADOS}

Durante este período se han notificado 128 situaciones conflictivas por parte de los 4842 trabajadores del área 6 de la Comunidad Autónoma de Madrid incluidos en el estudio, siendo la prevalencia de las notificaciones de situaciones conflictivas un 2,6\% (IC95 \% 2,2-3,1).

Los trabajadores de atención primaria notifican las situaciones conflictivas un $8 \%$ frente al 1,2\% de los trabajadores de atención especializada $(\mathrm{p}<0,001)$, encontrando diferencias significativas entre las notificaciones en los dos ámbitos. (Tabla 3)

En atención primaria los trabajadores de los centros sanitarios del ámbito rural notifican más las situaciones conflictivas (12,5\%), mientras que los trabajadores de los centros sanitarios del ámbito urbano notifican menos $(4 \%)(\mathrm{p}<0,001)$, encontrando diferencias entre ambos grupos. 
Tabla 3. Notificaciones por ámbitos de actuación y centros de trabajo

\begin{tabular}{lcr}
\hline & Atención primaria & \\
\hline & Urbano & \multicolumn{1}{c}{ Rural } \\
\hline Administrativo & $2(1,0 \%)$ & $19(21,6 \%)$ \\
Celador & $1(4,5 \%)$ & $3(17,6 \%)$ \\
Enfermeras & $7(3,5 \%)$ & $13(8,2 \%)$ \\
Facultativos & $14(6,4 \%)$ & $21(11,8 \%)$ \\
\hline TOTAL (p>0,001) & $24(4,3 \%)$ & $56(12,5 \%)$ \\
\hline
\end{tabular}

\begin{tabular}{lrr}
\hline \multicolumn{3}{c}{ Atención especializada } \\
\hline H.E. & H.U.P.H.M. \\
\hline Administrativo & $1(1,8 \%)$ & $10(5,6 \%)$ \\
Celador & - & $8(5,9 \%)$ \\
Enfermeras & - & $22(1,2 \%)$ \\
Facultativos & $1(1,0 \%)$ & $6(0,62 \%)$ \\
\hline TOTAL (p>0,001) & $2(0,42 \%)$ & $46(1,4 \%)$ \\
\hline
\end{tabular}

En atención especializada los trabajadores del Hospital de Guadarrama no han notificado durante este período ninguna situación conflictiva con los ciudadanos, frente al $0,42 \%$ de las notificaciones efectuadas por los trabajadores del Hospital del Escorial y el 1,4\% de los trabajadores del Hospital Universitario Puerta de Hierro Majadahonda.

Según las categorías laborales el personal administrativo es el que mas notifica $(7,4 \%)$, seguido de los celadores $(5,4 \%)$, facultativos $(2,8 \%)$ y enfermeras $(1,7 \%)$ (Tabla 4)

Los fisioterapeutas, los técnicos y los higienistas dentales no han notificado ninguna situación conflictiva en nuestro período de estudio.

Tabla 4. Notificaciones por categorías laborales entre AP y AE

\begin{tabular}{lrrrr} 
& \multicolumn{1}{c}{ AP } & \multicolumn{1}{c}{ AE } & \multicolumn{1}{c}{ TOTAL } & \multicolumn{1}{c}{ P } \\
\hline Facultativos & $396(8,8 \%)$ & $1082(0,6 \%)$ & $1478(2,8 \%)$ & $<0,001$ \\
Enfermeras & $342(5,8 \%)$ & $2158(1,0 \%)$ & $2500(1,7 \%)$ & $<0,001$ \\
Administrativos & $191(10,7 \%)$ & $244(4,7 \%)$ & $418(7,4 \%)$ & 0,019 \\
Celadores & $39(10,2 \%)$ & $182(4,3 \%)$ & $221(5,4 \%)$ & 0,28 \\
\hline
\end{tabular}

AE: atención especializada

AP: atención primaria

En función de las categorías laborales y del ámbito de actuación los facultativos de AP comunican un $8,8 \%$ frente al $0,6 \%$ de los de $\mathrm{AE} \quad(\mathrm{p}<0,001)$, encontrando diferencias estadísticamente significativas. Las enfermeras en AP notifican un 5,8\% en AP frente al $1 \%$ de $\operatorname{AE}(\mathrm{p}<0,001)$, encontrando diferencias significativas entre los dos grupos. Los administrativos de AP notifican un 10,7 \% frente al 4,7 \% de AE ( $\mathrm{p}=0,019)$, encontrando diferencias. Los celadores de AP notifican las situaciones conflictivas en AP (10,2\%) frente al $4,3 \%$ de los de $\mathrm{AE}(\mathrm{p}=0,28)$. El grupo de los celadores es la categoría laboral que no presenta diferencias estadísticamente significativas en la notificación por categorías entre AP y AE.

En el Hospital del Escorial ni los celadores ni el personal de enfermería han comunicado ningún tipo de incidencia.

De las 128 situaciones conflictivas notificadas por los trabajadores del área 6 incluidos en nuestro estudio han sido agresiones verbales (90,6 \%) IC 85,18-96,06, agresiones físicas $(9,4 \%)$ IC 3,93-14,81 encontrando diferencias estadísticamente significativas en ambos grupos. El 58,3\% de las agresiones físicas han presentado lesiones, 
frente al $10,3 \%$ de las agresiones verbales $(\mathrm{p}<0,001)$ con diferencia significativa. Han necesitado baja laboral un $8,3 \%$ de las agresiones físicas frente al 4,3\% de las agresiones verbales. $(\mathrm{p}=0,45)$; no encontrando diferencias estadísticamente significativas entre las bajas laborales ocurridas en agresiones físicas y verbales, por lo que no puede afirmarse que las agresiones físicas necesiten más bajas laborales que las agresiones verbales. Las agresiones físicas pueden acompañarse de agresiones verbales.

Han necesitando apoyo psicológico 7 trabajadores un 5,5\% de los trabajadores agredidos que notificaron el incidente.

\section{DISCUSIÓN/CONCLUSIÓN}

Los trabajadores de atención primaria del ámbito rural del área 6 de la CAM son los que más notificaciones de situaciones conflictivas realizan, seguidos de los trabajadores de los centros sanitarios del ámbito urbano, siendo los trabajadores del Hospital Puerta de Hierro los que más notifican en AE.

Los trabajadores del estudio notifican más las situaciones conflictivas en AP que en AE, encontrando diferencias significativas a excepción del grupo de los celadores en los que no puede afirmarse esta relación.

Por categorías laborales el personal administrativo, es el que más notificaciones realiza del área 6 de la CAM, siendo de mención la diferencia de notificación entre los facultativos de primaria y especializada.

De las notificaciones comunicadas la mayoría son agresiones verbales, presentando las agresiones físicas más lesiones que las verbales, pero no puede afirmarse que las agresiones físicas tengan más bajas laborales que las psíquicas

La incidencia de la notificación de las situaciones conflictivas con los ciudadanos en el área 6 de la CAM es de un 2,6 \%. Este resultado no es representativo de la situación real $^{1,2}$ ya que tenemos constancia de la existencia de muchos más incidentes que no se notifican adecuadamente. Se ha valorado el registro de intervenciones efectuadas por los servicios de seguridad privada y de las fuerzas de seguridad del estado y son superiores a la prevalencia de las notificaciones.

Las notificaciones en AP son más elevadas (8\%) que en $\mathrm{AE}(1,2 \%)$ creemos que en ello intervienen distintos factores, como puede ser la difusión del modelo y del procedimiento de actuación en el portal interno de atención primaria del área 6 al que tienen acceso todos los trabajadores. Otro factor condicionante podría ser la implicación directa de la gerencia en cada uno de las notificaciones, por el contacto directo con el trabajador afectado y seguimiento de la situación. ${ }^{9}$

Creemos que la falta de notificación en el Hospital de Guadarrama y en el Hospital del Escorial, podría deberse a que no ha existido una difusión adecuada del procedimiento, la mayoría de los trabajadores desconocen como notificarlas, y en caso de conocerlo, no lo notifican porque creen que no existe una implicación por parte de la gerencia, lo que justifica el 0,42\% en el Hospital del Escorial y la no comunicación de los incidentes en el Hospital de Guadarrama.

En AP los centros del ámbito rural (12,5 \%) comunican mucho más que los centros del ámbito urbano (4\%), debido a la existencia del SAR (Servicio de Atención Rural), trabajadores que realizan las guardias nocturnas y festivas con unas condiciones de trabajo distintas a los centros del ámbito urbano, siendo estos colectivos más susceptibles a las agresiones. A esto hay que añadir que en la zona rural hay determinadas zonas geográficas con una población marginal y problemas sociales con una mayor conflictividad que la media de la población. ${ }^{7}$

Es significativo respecto a categorías, la mayor notificación por parte de los facultativos de AP (8,8 \%) respecto a los de AE (0,6 \%), puede ser debido a la relación más 
directa y cercana que tienen los ciudadanos con sus médicos de familia, así como que estos gestionan la incapacidad temporal y la solicitud de recetas y pruebas complementarias.

Los administrativos que son el primer filtro en la recepción de los pacientes que llegan a los centros sanitarios puede justificar que sean los trabajadores que más notificaciones realicen (10,7\%), ya que sobre ellos los ciudadanos descargan su agresividad y frustración ante los problemas y las expectativas no cumplidas que se encuentran en los centros sanitarios.

No encontramos una justificación a la falta de notificaciones de situaciones conflictivas por parte de los fisioterapeutas, técnicos e higienistas dentales.

De las notificaciones comunicadas la mayoría son agresiones verbales (insultos, amenazas, coacciones) 90,6 \%, solamente en situaciones concretas, los ciudadanos agraden físicamente a los trabajadores sanitarios como puedan ser pacientes psiquiátricos o con problemas psicosociales. La mayoría de los ciudadanos utiliza la violencia verbal para demostrar su descontento al no cumplirse sus expectativas. ${ }^{5,6}$

Las diferencias significativas detectadas en las bajas laborales entre las agresiones físicas y las psíquicas, pueden deberse a que muchas agresiones verbales en función de la susceptibilidad del trabajador que las recibe puede terminar en problemas de ansiedad o psíquicas que conducen a una baja laboral.5,6,9

\section{AGRADECIMIENTOS}

Por su inestimable ayuda en el análisis epidemiológico y estadístico a:

Fernando García López. Unidad Epidemiología del Hospital Universitario Puerta de Hierro. Majadahonda (Madrid). España.

Isabel Millán Santos. Servicio de Bioestadística del Hospital Universitario Puerta de Hierro. Majadahonda (Madrid). España.

\section{REFERENCIAS BIBLIOGRÁFICAS}

1. OIT, OMS, CIE, ISP: Directrices Marco para afrontar la violencia laboral en el sector de la salud. Ginebra 2002.

2. OIT, OMS, CIE, ISP. Ops. Cit.

3. Nordin, H. Sistema de información sobre las lesiones en el trabajo. 1995.

4. Agencia Europea para la Seguridad y la Salud en el Trabajo. F. 24, La violencia en el trabajo. 2002.

5. Kristensen TS. Job stress and cardiovascular disease: a theoretic critical review. J Occup Health Psychol 1996.

6. Luna, M. Riesgos Psicosociales en los empleados de la Administración Pública en la Comunidad de Madrid. USMR. CCOO 2006.

7. Yela, C. Relación de los profesionales sanitarios y los pacientes en la violencia en el sector sanitario.

8. Di Martino, V. Violence at work. Ginebra: Internacional Labour Organization. 1998.

9. NTP 489-1998. Violencia en el lugar de trabajo. INSHT.

10. Quaderns de la bona praxi. Guia per preveni i gestionar la violència en el lloc de treball contra els metges. Col.legi Oficial de Metges de Barcelona. 2004.

11. Orden 212/2004, de 4 de marzo, del consejero de Sanidad y Consumo, por las que se establecen las directrices y líneas generales para la elaboración de planes de prevención y atención frente a potenciales situaciones conflictivas con los ciudadanos en los centros e instituciones sanitarias públicas.

12. Orden 683/2006, de 24 de marzo de la Consejería de Sanidad y Consumo, por la que se modifica la Orden 212/2004 de 4 de marzo de la Consejería de Sanidad. 
13. Orden $22 / 2008$, de 21 de enero, se crea el fichero de datos de carácter personal denominado plan regional de Prevención de situaciones conflictivas dependiente de la Dirección General de RRHH de la Consejería de Sanidad de la Comunidad de Madrid.

14. Resolución de la Dirección General de Recursos Humanos de 29 de enero de 2009, se dictan instrucciones para la puesta en marcha del Registro Centralizado de la Consejería de Sanidad para Situaciones conflictivas con los ciudadanos.

15. Violencia hacia el profesional de la salud: prevención, manejo y actuación post incidente. Creación de un registro de notificación por Internet en Cataluña. Genís Cervantes i Ortega.

16. Aggression Towads Health Care Workers in Spain: A Multi-facility Study to Evaluate the Distribution of Growing Violence Among Professionals, Health Facilitgies and Departaments (Gascón, S: MartínezJarreta, B; Santed, M.A: González Andrade, J.F. y Casolod, Y) International Journal of Occupational and Environmetal Health (Volumen 15, n. ${ }^{\circ} 1$ [2009]).

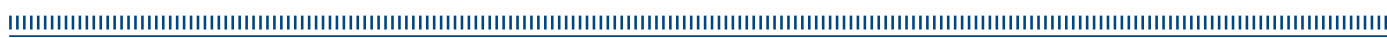




\section{ANEXO I: MODELO DE NOTIFICACIÓN}

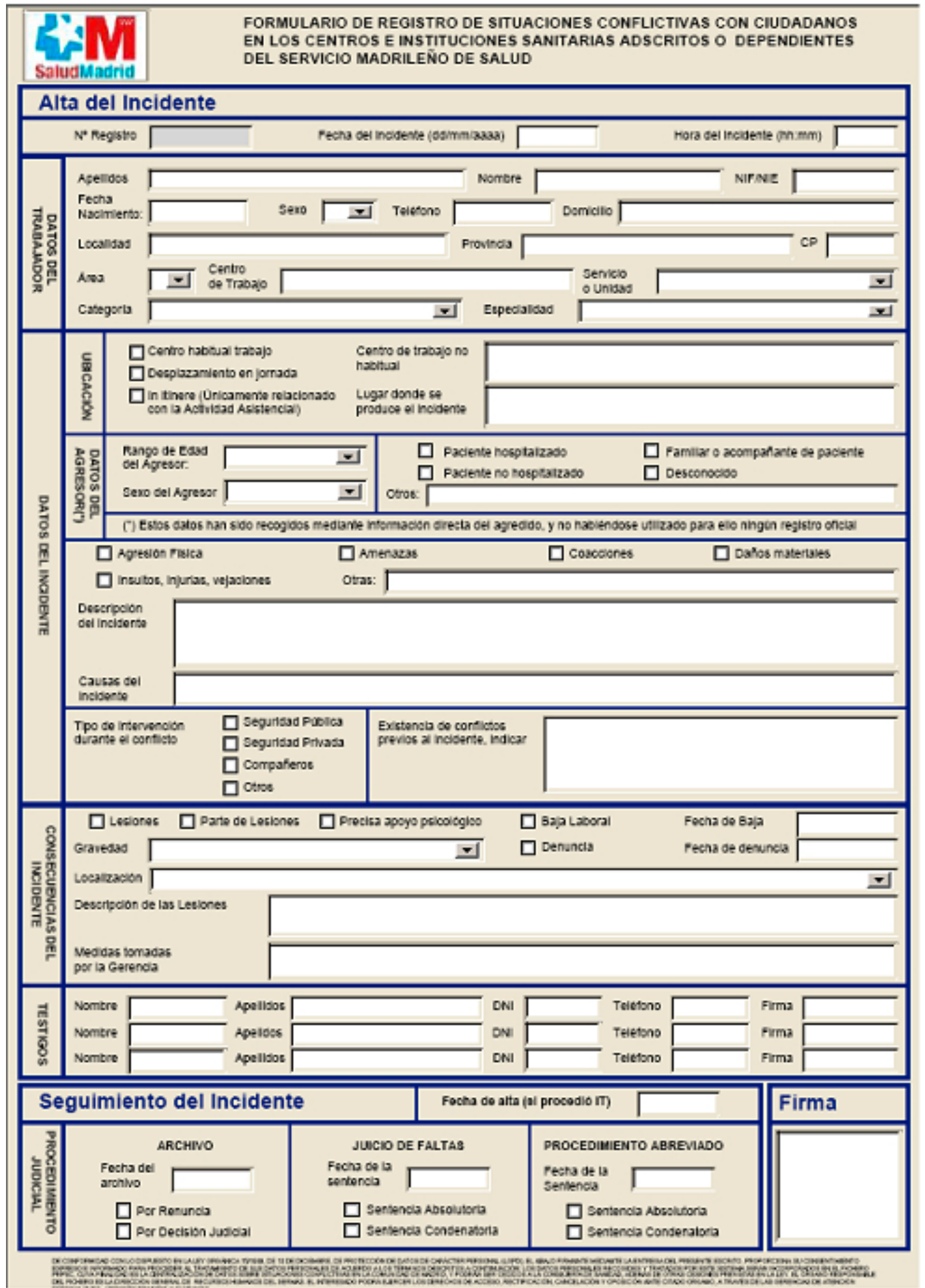




\section{ANEXO II: ÁREAS SANITARIAS DE LA COMUNIDAD AUTÓNOMA DE MADRID}

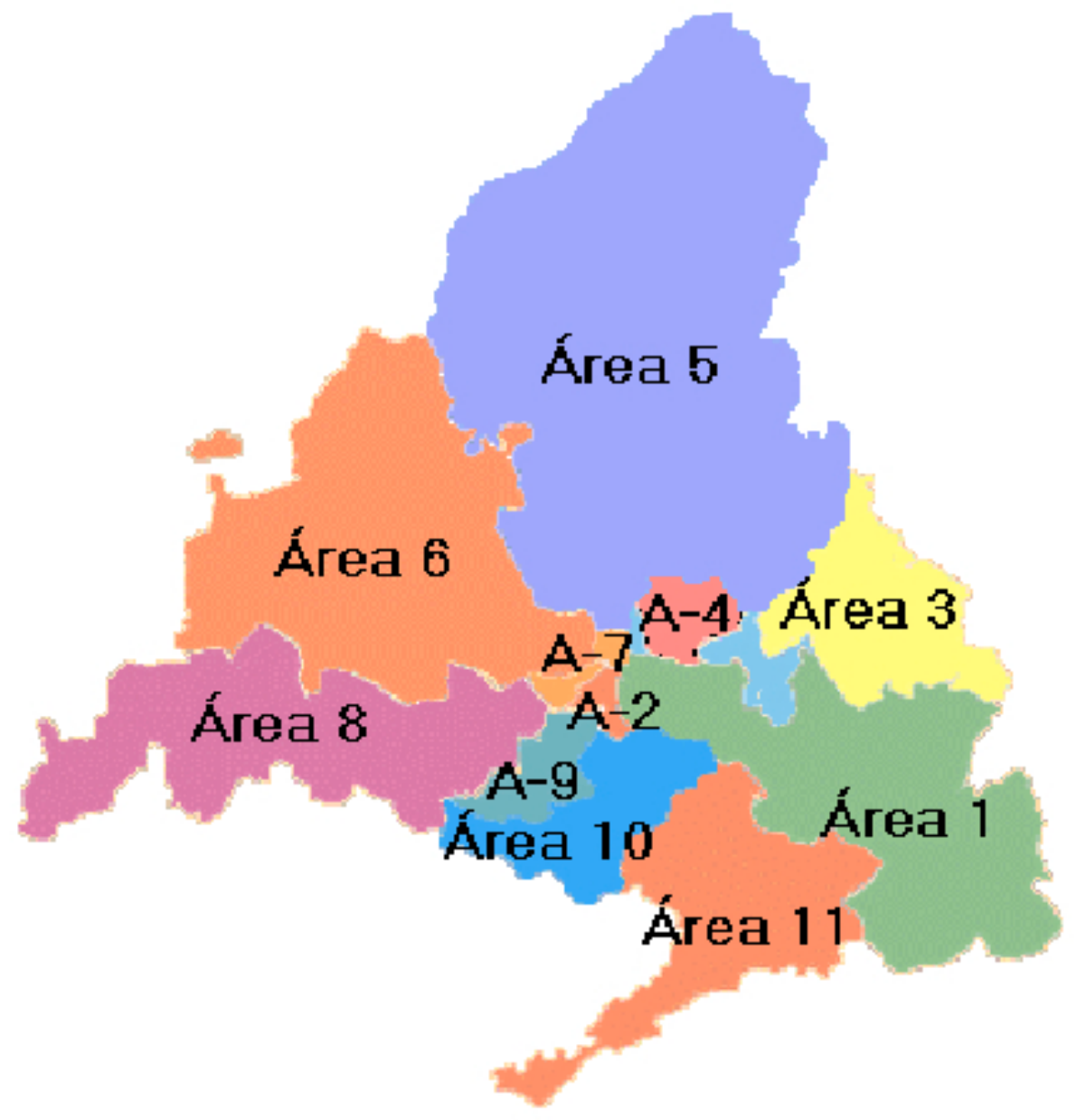

